\title{
Autoritarismo, resistencia y acoso laboral en la academia del siglo XXI: rostros inuevos? de una vieja exclusión \\ Authoritarianism, resistance and mobbing in the 21st century academy: New? faces of an old exclusion
}

Amparo Saornil Comaposada

Investigadora independiente - España

Madrid, España

amparo.saornil.comaposada@protonmail.com

\section{RESUMEN}

El presente artículo tiene lugar a partir de un estudio de caso autoetnográfico basado en experiencia de acoso laboral de la autora en una universidad española. El objetivo central del trabajo es examinar, desde un abordaje de ética aplicada, la compleja trama de poder en la que emergen y se desarrollan prácticas de violencia y acoso laboral en instituciones académicas y universitarias. Frente a los dispositivos que facilitan la perpetuación de estas prácticas de violencia y exclusión, así como su naturalización, el artículo propone revisitarlas a la luz de los criterios de las éticas organizacionales y los liderazgos morales, en aras de enriquecer las herramientas ya existentes en distintas universidades para detectar acoso y promover éticas colectivas. El artículo argumenta la necesidad de potenciar las estrategias multimodales contra el acoso laboral en la academia, a fin de que realmente sean efectivas y no parte de protocolos meramente formales. Finalmente, se propone apelar a una profunda crítica desde la ética filosófica en torno a los discursos y prácticas de acoso laboral académico y universitario, para erradicar las violencias no sólo en estas instituciones sino en cualquier ámbito de la vida social.

Palabras clave: acoso laboral; endogamia; universidad; autoetnografía; ética organizacional

\section{ABSTRACT}

This article arises from an autoethnographic case study based on the author's experience of harassment at work in a Spanish university. The main objective is to examine from an approach of applied ethics, the complex web of power in which emerge and develop practices of violence and harassment in academic and university institutions. Against the dispositive that facilitate the perpetuation of these practices of violence and exclusion, as well as their naturalization; the article proposes to revisit them in the light of the criteria of organizational ethics and moral leaderships, to enrich the tools already existing in different universities to detect mobbing and promote collective ethics of others. The article shows and argues the need to strengthen and radicalize the multimodal strategies to eradicate mobbing in the academy and the university so that they are effective and not part of merely formal protocols. Finally, it proposes to appeal to a deep criticism from the philosophy and ethics around the speeches and practices of academic and university mobbing to eradicate violence not only in these institutions but in any area of social life.

Keywords: mobbing; endogamy; university; autoethnography; organizational ethics 


\section{o. Proemio. Las tres maldiciones imperdonables en el acoso laboral: iAvada Kedavra!}

Barty Crouch Jr. (como Alastor Moody): “Pero primero, ¿quién puede decirme cuántos Maleficios Imperdonables existen?" Hermione Granger: "Tres, señor”.

Barty Crouch Jr. (como Alastor Moody): “¿Y por qué se llaman así?” Hermione Granger: “Porque son imperdonables. Si un mago los usara...”. Barty Crouch Jr. (como Alastor Moody): "Se ganaría un boleto directo a Azkaban, icorrecto! El Ministerio dice que son muy jóvenes para ver sus efectos, ipero yo opino lo contrario! iiNecesitan saber a qué se enfrentan!! iiiY necesitan estar preparados!!!” (Clase de Defensa Contra las Artes Oscuras de $4^{\circ}$ año en Hogwarts, 1994')

En un lugar cruzando Despeñaperros (quedará para el misterio si subiendo o bajando), de cuyo nombre no quiero acordarme, había una universidad donde acaecieron, no mucho tiempo ha, los sucesos que relataré, con la mayor fidelidad a la verdad. Las maldiciones (o maleficios) imperdonables son tres de los hechizos más poderosos y siniestros conocidos en el mundo mágico de Harry Potter; herramientas de las Artes Oscuras, fueron clasificadas por primera vez como "imperdonables" en 1717, con las sanciones estrictas asociadas a su uso.

Estos maleficios tan imperdonables son, por orden creciente de gravedad: 1) la Maldición Imperius (“ilmperio!"), que somete la voluntad de la persona sobre la que recae a la voluntad del mago o bruja ejecutor; 2) la Maldición Cruciatus (“iCrucio!”), que consiste en la tortura del ejecutor sobre otra persona (suele subseguir al mandato de "Imperio", si la obediencia no ha funcionado convenientemente); 3) la "Maldición Asesina”, joya de la corona de las maldiciones imperdonables y de la magia negra, que se lanza al espeluznante grito de “iAvada Kedavra!" y consiste, como es evidente, en la aniquilación física y definitiva de la persona sobre la que recae.

Resulta muy curioso, por cierto, que en este formidable universo mágico se considere imperdonable no solo la muerte sino, también, la tortura y, sobre todo, el sometimiento de la propia voluntad para convertirse en marioneta de otro ser.

Pues bien, salvando las distancias, encontramos que estas maldiciones imperdonables retratan simbólicamente, casi a la perfección, el proceso como sucede un acoso laboral en la forma en suele desarrollarse en la universidad contemporánea, ligado a la endogamia, la obediencia y el vasallaje y, sobre todo, a lo que subsigue si hay una disrupción o ruptura de estos. Así, en primer lugar se emite un Imperio: la persona con poder espera la obediencia del súbdito o, en lenguaje del siglo XXI, subordinado o subalterno (ya sea doctorando, profesor-a más novato, en fase de consolidación, etc.) - en mi título, "autoritarismo"-; si se desobedece o se desacata en alguna forma ("resistencia"), entonces se lanza un Crucio: comienza la tortura, no en una forma física, claro, pero sí en la vía (o, mejor, viacrucis para el caso que nos ocupa) del mobbing ${ }^{2}$, bullying adulto en realidad como se ha dado en llamar: hacer "la vida imposible" a la persona desobediente, buscando al fin lo que se llama en teoría del acoso laboral la "muerte institucional", esto es, que la persona acosada salga de la institución, bien porque se logre su expulsión formal, bien porque acabe por irse por su propio pie, desesperada (opción que supone el logro más alto para la persona acosadora, ya que implica, por ejemplo en contextos empresariales, que no se considere despido improcedente y por tanto no haya finiquito, es decir, coste alguno para el empleador o líder). No es infrecuente el suicidio. Et voilà!: tenemos así un Avada Kedavra de libro que, además, resulta el crimen perfecto (como se ha denominado también al mobbing): no deja huellas.

Estas tres maldiciones (Imperio, Crucio, Avada Kedavra) son imperdonables porque vulneran la dignidad humana de modo fundamental y difícilmente reparable, o directamente imposible en el caso de la aniquilación física. Están castigadas con cárcel perpetua en Azkaban (la terrible prisión del mundo mágico), vigilada por dementores ciegos, unas criaturas oscuras que roban toda forma de alegría.

1 Extraído de: https://cutt.ly/Uh3GQe7 Se trata de la reproducción del diálogo correspondiente en la película "Harry Potter y el Cáliz de Fuego", basada en el libro homónimo (cuarta entrega de la saga) de J.K. Rowling.

2 Tanto para este término inglés como para otros similares, se indica sin cursiva por sus altos uso y aceptación en la literatura en castellano. 
Para mí, autora de este texto y protagonista de la autoetnografía que lo motiva a raíz de un acoso laboral (en ámbito universitario vinculado a ciencias humanas y sociales), estos espacios terribles han constituido aquellos otros no-lugares de la política contemporánea, donde también, o muy especialmente, se juegan la ética y la política, en el mismo intestino de lo que producimos.

Denuncio así la incongruencia de que los valores predicados desde palestras en ciencias humanas y sociales para subvertir el racismo, el sexismo, el clasismo, etc., (en suma, toda forma de discriminación), no se apliquen en las distancias cortas de las relaciones intradepartamentales, reproduciéndose en cambio el vasallaje propio del feudalismo premoderno; ello se denota evidentemente en su versión contemporánea de la desigualdad neoliberal, inequidad que se reproduce sin ambages ni recato en las bambalinas departamentales. Dicho de otro modo, el mismo ejercicio de poder que no se considera ético, moral, lícito, en otros ámbitos, que además se singulariza en su denuncia especialmente desde las ciencias sociales y humanas (en pro de lo decolonial, entre otros), se aplica sin rubor en esa inercia de la universidad, del respeto -o mejor, temor- y el acatamiento de las jerarquías tácitas de los feudos departamentales. Y, por supuesto, la condena y la persecución cuando se desobedece: el acoso laboral, el daño ejercidos contra mí en el caso que nos ocupa, nació y se desarrolló precisamente al calor de tal abierto desafío.

En definitiva, a menudo es la academia, paradójicamente, el último bastión del conservadurismo en la forma del rostro más rancio del clientelismo académico (por no decir abierto nepotismo), donde se justifica lo que no valdría en ningún otro espacio ciudadano o arena política, desde el cinismo más basal. Así, las desigualdades estructurales devienen fundamentales aquí, cifradas en el sentido de la tribu clientelar más ancestral o, dicho de otro modo, el sistema de lealtades feudal, de vasallaje no basado en meritocracia horizontal sino en el intercambio de prebendas vertical, donde la jerarquía estructura y vehicula aquel sistema de cooptación y nepotismo clásico.

Este artículo se enmarca plenamente, pues, en el objetivo de analizar formas diversas de autoritarismo y sus resistencias, de articulación de luchas y protestas sociales contra formas de poder ejercidas en los lugares paradigmáticos donde se producen las teorías contra el poder. Se reflexionará sobre la transversalidad del movimiento contestatario y de resistencias frente al neoliberalismo como proyecto integral (económico, político y cultural) que busca perpetuar el dominio del proyecto capitalista (patriarcal-colonial-moderno). Se tratará, así, de uno de los últimos tabúes (si no el último) para hablar en una palestra científica: la corrupción endogámica en la reproducción de la vida académica y su subversión, en la forma de una lucha, con la articulación de diferentes modos (administrativo, legislativo y sindicalista-libertario) de contestación a una injusticia en los intestinos mismos de un departamento universitario al sur de Europa.

\section{Introducción: vislumbrando el acoso laboral}

Para esto no merece la pena que haya dios.

(Caín, José Saramago)

El acoso laboral o mobbing es ya considerado como la nueva plaga laboral del siglo XXI y una auténtica epidemia organizativa, en la que por desgracia destaca su presencia especialmente en el ámbito universitario (Piñuel y Zabala, 2001, p. 50). A menudo implica consecuencias más negativas (efectos más graves y duraderos) para las víctimas que el propio acoso sexual (Hershcovis et al, 2015, p. 2015).

Es fundamental distinguir entre los conflictos, que inevitablemente suceden entre personas en cualquier circunstancia, y el acoso propiamente dicho, como precisa uno de los estudiosos pioneros de la cuestión:

Nos referimos a un tipo de situación comunicativa que amenaza con infligir al individuo graves perjuicios psíquicos y físicos. El mobbing es un proceso de destrucción; se compone de una serie de actuaciones hostiles que, tomadas de forma aislada, podrían parecer anodinas, pero cuya repetición constante tiene efectos perniciosos (Leymann, 1996, p. 26).

De hecho, repárese también en que "Las estrategias utilizadas para someter a la víctima al psicoterror son muy variadas y la mayor parte de las veces se combinan unas con otras a modo de "tratamiento integral" (Leymann, 1996, p. 26). 
Como consideración de índole terminológica, los términos más populares en la literatura para referir al fenómeno de esta patología en el lugar de trabajo son "mobbing" (más habitual en fuentes polacas y escandinavas) y "bullying" (muy frecuente en el inglés) (Nadja, 2013). Otras denominaciones de cierta incidencia son "intimidación psicológica" y "asedio moral" o "psicológico". Aunque existen algunos matices interpretativos para cada término, los investigadores los usan de forma intercambiable (Nadja, 2013). Aquí se usará indistintamente "acoso laboral" o el inglés "mobbing" (por su generalizado uso en castellano) y en algunos momentos, por economía lingüística y legibilidad del texto, simplemente "acoso" (refiriéndome pues siempre a su aceptación "laboral", nunca a otras formas de acoso como pueda ser el sexual).

Este artículo plantea una conjunción de revisión de estado de la cuestión y análisis bibliográfico (epígrafe 3 de marco teórico, construido con una metodología clásica de revisión de base de datos y su correspondiente heurística, con énfasis en el enfoque antropológico-filosófico, en congruencia con mi formación y perfil) y estudio de caso (epígrafe 4), en la forma de una autoetnografía a raíz de mi experiencia de acoso laboral en una universidad española. En el epígrafe metodológico (2) se aportará bibliografía contemporánea referencial sobre la autorización de la autoetnografía precisamente para casos de investigadores con experiencia propia en padecimiento de acoso laboral. Finalmente, se pergeña una reflexión y una discusión de los datos, combinando los de segunda mano con los propios de primera mano, para clausurar con una advocación y una prospectiva críticas y propositivas sobre esta forma de daño y discriminación específicos del ámbito académico, crucial, habitual por desgracia, muy vieja aunque se le proporcione rostros nuevos, y que sin duda debe ser nombrada, politizada y combatida con estrategias multimodales.

\section{Método autoetnográfico: personalizando el acoso laboral}

El método usado para la elaboración de la base empírica de esta investigación es la etnografía y, dentro de la misma, más concretamente, la autoetnografía, siendo las técnicas la observación participante y las conversaciones abiertas por un espacio de casi una década, desde 2011 hasta el presente. Para ello, me he inspirado en notables trabajos previos elaborados desde estas palestras metodológicas. Así, Allison et al (2011, p. 4) destacan los siguientes valores de la autoetnografía aplicada a este tipo de estudios: "We can level the ground between the "high ground" of the academic community and the "swampy lowlands" of practice; producing knowledge and enabling exchange between communities" (ver también Akella, 2016).

Resulta interesante cómo destaca la presencia de autoetnografías de profesionales y académicos en este ámbito de estudios, seguramente porque las personas en situaciones de este tipo se hallan de hecho en una posición privilegiada -paradójicamente- para pensar sobre un fenómeno de tan difícil abordaje, desde un lugar no solo encarnado sino, también, absolutamente autorizado y legitimado por la propia experiencia biográfica, cubriéndose esos huecos que señalan Allison et al (2011) entre el "alto nivel” de la comunidad académica y los "bajos pantanos" en la práctica real de la gente de carne y hueso (cf. también Deckers, 2020).

En la misma estela escribe Murphy al respecto de la justicia y el coraje de hacer autoetnografías en situaciones de exclusión, al mostrarse la propia vulnerabilidad y vindicando el poder curativo de la narración:

One of the defining features of autoethnography is its ability to give a voice to those silenced or marginalized by those who are more powerful. When ethics determine that the perpetrator must be protected, the power remains with the perpetrator and the victim continues to be silenced. It is my contention then that to deny an individual the opportunity to explore their own story is in itself an act of oppression (Murphy, 2008, p. 166).

Destaca sin duda el trabajo de Pheko (2018), elaborado a través de una autoetnografía que investiga su experiencia personal de acoso laboral en la universidad, usando la escritura como terapia, la adaptación cognitiva y la descripción de sus propias estrategias de dotación de sentido a la vida empleadas por esta académica botswanesa para sobrevivir. Como describe Pheko, en mi propio caso igualmente la historia personal sirve a la vez de conjunto/fuente de datos, como método de investigación y para la aplicación de la técnica de escrita terapéutica, dada la gran afectación que implica el acoso. Como señala también Pheko (2018), este encarnamiento (aunque ella no aplique el término concreto) supone un fácil acceso a todos 
los elementos más y menos duros de esta experiencia singular: desde el crudo dolor más íntimo hasta los detalles administrativos más prosaicos. Así, la primera persona ayuda a minimizar precisamente los huecos (cf. Allison et al, 2011) en la investigación de este fenómeno tan complejo como delicado.

Como reflexión deontológica al respecto del anonimato del departamento como entorno colaborador y la propia persona acosadora (como veremos, sancionada ahora formalmente por "falta muy grave de acoso laboral” según la administración española), en ningún momento se mencionará nombres reales (ya sea de institución o de persona), incluyéndome a mí como autora, y ello a pesar de que hay precedentes de estudios al respecto donde, en virtud del derecho a la información, sí se mencione nombre de instituciones -no de personas concretas (Lee, 2019; Muncey, 2005). El motivo principal y preclaro de este anonimato es mi autoprotección como víctima del acoso.

Por otro lado, todas las afirmaciones sobre situaciones de conflicto, sobre las que pudiera criticarse parcialidad, son defendidas y expresadas como hechos probados en tanto que se trata de tales: no se trata opiniones a raíz de una contienda personal sino que se basan en evidencias intersubjetivamente cotejadas durante la instrucción del caso, probada ya la estructura discriminatoria, y en tanto que ha existido ya una investigación finalizada en sanción sobre el caso que nos ocupa, como se explicitará en el estudio de caso.

Con todo, se ha de tener en cuenta que este artículo forma parte de una investigación más detenida y compleja en curso (work-in-progress), que incluye el abordaje de otros casos análogos y un mayor desarrollo de la propia autoetnografía, en elaboración, de la que aquí se destacará solamente los elementos más relevantes hasta ahora trabajados.

\section{Marco teórico-conceptual: tematizando el acoso laboral}

\subsection{A modo de previo: complejizando la hermenéutica de una noción vieja y nueva}

Lo que hoy llamamos "acoso laboral", según sus mayores especialistas, es algo tan arcaico como el ser humano. Piñuel y Zabala describe al Cid Campeador ${ }^{3}$, por ejemplo, como una víctima clásica de acoso laboral en su contexto histórico. Con todo, esta antigüedad no obsta la necesidad de abordaje detenido en la forma específica que ha adoptado en el mundo contemporáneo este modo singular de hostigamiento, discriminación, persecución y, al fin, ejercicio de daño desigual entre seres humanos que, entre otras denominaciones, está muy próxima a la definición del proverbial “cainismo”.

El mobbing puede darse en estructuras organizacionales y de modos muy heterogéneos: empresas, entornos sanitarios, centros de educación de diverso nivel (entre ellos, las universidades, etc.). Ello ha dado lugar en los últimos años a una nutridísima bibliografía que abarca desde lo jurídico -la necesidad imperiosa de catalogar, describir, tipificar en lo jurídico en una inmensa mayoría de sistemas judiciales que ni siquiera contemplaban esta figura hasta hace pocos años-, hasta lo psicológico -la urgencia de desarrollar e implementar instrumentos afinados de medida, detección, peritación, etc., así como su prospectiva y sus soluciones-, pasando por lo sanitario, lo sociológico e incluso lo neurológico (las relaciones íntimas, por ejemplo, del mobbing con el síndrome del burnout).

Las reflexiones a colación de tipo más antropológico y moral-filosófico son sin embargo, todavía, escasas o casi inexistentes. Así, se pone de manifiesto la imperiosidad de articular cada vez más el conocimiento ya desarrollado en mayor medida por otros ámbitos de saber diferentes, para ir creando un ámbito propio de las humanidades y las ciencias sociales sobre esta forma de acoso, del mismo modo que existe ya un tupido conocimiento desarrollado al respecto desde tales campos de saber sobre el acoso sexual, por ejemplo.

Se presenta aquí una revisión narrativa de los principales hallazgos de los últimos años en disciplinas estudiosas del mobbing, de un lado, y en enfoques complementarios como los de la filosofía moral sobre el liderazgo y la ética organizacional, de otro, para pergeñar una reflexión antropológico-filosófica sobre la cuestión orientada especialmente a la academia, y en tanto que se ha detectado que este tipo de trabajos brillan todavía por su ausencia en el campo de estudios específico sobre el acoso laboral.

Los principales buscadores empleados han sido ResearchGate, AcademiaEdu, Google Scholar, Philosopher Index, Latindex, Dialnet. Los términos primarios de búsqueda, en castellano y en inglés, ha sido "acoso

3 Cf. http://acosopsicologico.com/acoso-en-el-trabajo/videos/ 
laboral" y "mobbing", derivándose a otros relacionados en función de los hallazgos. Igualmente, se ha usado revisiones sistemáticas ya existentes que se citará oportunamente.

Para esta revisión se ha considerado estudios cuantitativos y cualitativos, tanto de tipo más genérico sobre acoso en organizaciones diversas y empresas, como específicos sobre acoso en instituciones de enseñanza en general y universidades en particular.

\subsection{Principales enfoques y estado de la cuestión: acotando el acoso laboral como problema ético y de salud pública global}

El acoso laboral es considerado hoy uno de los fenómenos más estresantes (Pheko, 2018; Hershcovis, 2008 y Hershcovis et al, 2015; Leymann, 1996; Piñuel y Zabala, 2001; Akella, 2016; Björklund et al, 2019; Bakan et al, 2017; Kowal et al, 2019; Stouten et al, 2010), con efectos más dramáticos en detrimento de la salud y la seguridad personales (González et al, 2008) y de mayor impacto negativo como comportamiento desviado, en definitiva, en la experiencia laboral y la salud personal (Stouten et al, 2010). Su importancia social está fuera de toda controversia y ha adquirido una centralidad radical en los estudios de la última década desde disciplinas, enfoques, metodologías y aplicaciones muy diversas, significando ya un marco de interés global que está constituido como un campo de estudios autónomo (Stouten et al, 2010; Björklund et al, 2019; Kowal et al, 2019).

Destacan estudios generales referenciales sobre el tema (Hershcovis, 2008 y Hershcovis et al, 2015; Leymann, 1996; Piñuel y Zabala, 2001) sobre mobbing en relación al liderazgo y la organización, ligándolo a la necesaria dimensión ética de las organizaciones contemporáneas (Akella, 2016; Björklund et al, 2019; Bakan et al, 2017; Kowal et al, 2019; Stouten et al, 2010; Harvey et al, 2009). Sobre su medición, destaca la elaboración de escalas diversas como la llamada "Cisneros" (Piñuel y Zabala y Fidalgo, 2004) o la de Rosander y Blomberg (2019). Y, finalmente, se ha tratado también sobre el mobbing específico en la universidad.

Concretamente acerca de este último, la gravedad y la prevalencia del acoso laboral en la universidad (García Landa, 2007; Justicia et al, 2003 y 2005; Rojas-Solís et al, 2019; Soares et al, 2020), una de las formas más sibilinas que adquiere la violencia estructural de la academia, no solo han merecido un interés creciente en los últimos años en la bibliografía jurídica (para su detección y tratamiento legislativo) (González et al, 2008) o psicológica (para su asistencia), sino también desde la perspectiva de la ética aplicada (con destacadas experiencias autoetnográficas) (Pheko, 2018; Allison et al, 2011), concretamente en los abordajes de lo que se ha dado en llamar estudios de (mal) liderazgo, análisis cruciales que describen cómo estas situaciones de ejercicio jerárquico del poder desarrollan experiencias, dinámicas y relaciones totalmente congruentes con el acoso laboral.

Se concuerda, así, en afirmar que el contexto de trabajo en la universidad presenta condiciones individuales y organizacionales singulares que favorecen especialmente el posible desarrollo del acoso psicológico (Parra y Acosta, 2010). Por otro lado, algunas autoras señalan que, comparativamente a otros campos de análisis de mobbing, sus correlatos en la universidad son todavía escasos (Björklund et al, 2019, p. 15).

Tan importante es trabajar en la prevención, la detección y la sanción -sucedido ya el daño-, como en la comprensión del fenómeno para, sobre todo, poder elaborar prospectivas adecuadas y generar atmósferas colectivas que, al fin, impidan el proceso de mobbing. Así, han sido descritos como predictores clásicos de mobbing factores como la falta de apoyo, el escaso control, la inconsistencia en las demandas de roles, el liderazgo pobre ( $y$, en función de ésta, el clima organizacional pobre) y el alto nivel de enfermedad (Björklund et al, 2020, pp. 11ss). La precariedad o inseguridad laboral es igualmente clave como predictor; sucede en numerosos nichos laborales, desde la empresa hasta la administración, pero de modo singular en la estructura académica/universidad, donde las situaciones ahora a menudo llamadas "en fase de consolidación", pendientes de decisiones externas (cooptadas por grupos de poder) durante años, inducen o bien a la sumisión para asegurar el puesto (seguridad) o bien, si se opta por la rebeldía/ contestación (libertad), tener que "pagar las consecuencias" de una hostilidad que deriva fácilmente en conductas de mobbing. Ese clásico dilema entre la seguridad y la libertad, proverbial en sus descripciones en ciencias sociales y humanas, adopta una especial pertinencia en el mobbing en la universidad.

En esta coyuntura, Soares et al (2020), en su estudio de caso en la Universidad Estatal brasileña, muestran también cómo la posgraduación supone un contexto fértil para el acoso por las presiones intensas de 
productivismo y alta competitividad, generando una afectación importante para la salud. Otros elementos cruciales descritos en y para situaciones de mobbing son la falta de claridad en roles y expectativas, la preexistencia de conflictos en el grupo donde la víctima es recién llegada, o las reorganizaciones de estructura. Estos factores permiten que el proceso de acoso continúe ya que favorecen la pasividad en los líderes e incluso, a menudo, que directamente los acosadores reciban apoyo de dichos líderes o jefes. A menudo dejar la organización es la salida preferida por las víctimas ${ }^{4}$.

Para el caso específico del mobbing en la universidad española, sus muy singulares maneras de hacer y de incidir en las víctimas, destaca el estudio de García Landa (2007) presentado con perspectivas perspectivas plurales, desde psicológicas a sindicales, pasando por un análisis meticuloso y exhaustivo de los intestinos académicos en su funcionamiento pseudofeudal disfrazado de siglo XXI. Igualmente importantes son los trabajos de Justicia et al (2003, 2005) para la Universidad de Granada (España), que arrojaron el resultado de que una de cada cuatro personas trabajadoras de dicha institución que participaron en su estudio se consideraban víctimas de acoso laboral, afirmando más de la mitad haber presenciado alguna vez situaciones de acoso en su trabajo. Se percibía más acoso en las mujeres y, en cuanto a los efectos negativos en salud de todo tipo y carrera profesional, destacaban muy especialmente el ámbito de las relaciones sociales de las víctimas como el aspecto más afectado a largo plazo.

Más en profundidad, se ha analizado una revisión sistemática muy reciente (Rojas-Solís et al, 2019), que cotejó a su vez estudios con enfoque cuantitativo y mixto, con diseño no experimental y transversal con un alcance correlacional y descriptivo. Sobre los instrumentos, se destacó la necesidad de la actualización teórica y la validación, por un lado, y de innovación en la medición, por otro.

Aunque pueda resultar baladí, han sido necesarios estudios cuantitativos y cualitativos intensos y numerosos para probar lo que pueda parecer una trivialidad: que existe una correlación positiva entre un liderazgo ético (apoyo de los altos mandos a comportamientos éticos, obligatoriedades morales y éticas de los jefes-Björklund et al, 2020, pp. 11ss-) y la satisfacción/salud laboral de las personas (Bakan et al, 2017). Así, la mayoría de los estudios concuerdan, por diversas vías y con métodos diversos, en la importancia del liderazgo, un liderazgo ético fuerte ya que su ausencia, lejos de permitir que florezcan círculos virtuosos de relaciones laborales, suele provocar la prevalencia e incidencia de los círculos viciosos de las viejas relaciones feudales donde el más fuerte -por antigüedad, por tradición, etc.- prevalece de modo particular (Björklund et al, 2019), hasta el punto de que se ha estudiado y descrito ya directamente el acoso como un "problema de gestión ética" (Kowan et al, 2018). Stouten et al (2010: 23) reconocen así también el rol central del liderazgo ético para atajar los comportamientos desviados estresantes para las personas trabajadoras.

Se ha descrito igualmente cómo ocurre lo contrario cuanto más fuerte es la cultura ética de una organización (Bakan et al, 2017); es decir, como sucede en tantos otros procesos sociales, se genera círculos bien virtuosos o bien viciosos, según sea el clima (in) ético de la atmósfera colectiva. Así, los expertos recomiendan con énfasis incrementar el clima ético de las organizaciones para elevar la satisfacción laboral de los empleados, y que se limiten o desaparezcan los comportamientos acosadores en empresas e instituciones (Bakan et al, 2017).

El mobbing necesita siempre un caldo de cultivo y una atmósfera favorable al mismo, de manera que, si bien suele haber una primera persona perpetradora, existe un coro alrededor que lo hace posible y es corresponsable en menor medida -o igual en algunos casos-, lo que en la literatura especializada ha llegado incluso a la acuñación de términos específicos. Así, la bibliografía más revisada hoy sobre acoso concuerda en referir a la estructura elemental tripartita de estos procesos, en los siguientes términos:

- Perpetradores (en inglés "perpetrators", victimarios o acosadores), se llama a las personas que ejecutan el acoso de forma directa, en primera persona (Björklund et al, 2019, pp. 7ss).

- Transeúntes (en inglés “bystanders”; Björklund et al, 2019, pp. 9ss) o cooperadores/colaboradores silenciosos necesarios, se llama a quienes están en el entorno del acoso y del acosador, favorables

4 No es baladí el dato de que "En un 90\% de los casos el acoso suele terminar con la salida de la persona de la organización [...]" (Piñuel y Zabala, 2001, p. 57). 
a este, por omisión a menudo o ayudando a extender rumores o cotilleos.

- Víctimas, incluso o chivos expiatorios (“scapegoating”, en inglés; Björklund et al, 2019, pp. 9ss) se llama a las personas acosadas.

Sobre el perfil de las víctimas, la literatura recoge cómo no son débiles psicológicamente o socialmente aisladas; antes bien, los estudios muestran que a menudo son personas de alta cualificación, muy compromitadas con el trabajo y con una alta capacidad para el mismo, así como un grado notorio de autonciencia; con frecuencia, más y mejor formadas, internacionalmente, etc., que los superiores donde se originan las actitudes de mobbing, cuyo germen no es ajeno al sentimiento de la envidia y de amenaza por el perfil superior de las víctimas estructuralmente subalternas. En cuanto al género, estas son mayoriatiramente mujeres según este estudio, en una diferencia de hasta un 70/30 por ciento (Björklund et al, 2019).

Giacalone et al (2016) han desarrollado la Teoría del Impacto Ético sobre cómo el comportamiento inético en el trabajo afecta al bienestar individual de forma radical. Su enfoque posee implicaciones cruciales en políticas públicas, porque el impacto deletéreo del comportamiento inético no se restringe solo a los individuos concretos involucrados sino que afecta a la globalidad: agentes externos, comunidades locales, instituciones, empresas, inversores, etc., hasta el punto de que el mobbing es descrito como un problema de salud pública (Giacalone et al, 2016). De hecho, las consecuencias (físico-psicosomáticas, sociales y económicas) para las familias de las víctimas y para las instituciones en general han sido ampliamente descritas (Kowal et al, 2018) (victimización terciaria). Así, se defiende cómo el trauma-estrés asociado con el comportamiento inético puede afectar al bienestar de otros a través de un proceso de traumatización secundaria y, en esa medida, en efecto se debe hablar de la implicación de estos estudios en políticas públicas (Kowal et al, 2018, p. 13).

\section{Estudio de caso5: politizando el acoso laboral}

\subsection{Líneas generales de un largo hostigamiento: telegrafiando el acoso laboral}

La relevancia de la experiencia que se narra aquí a modo de caso notorio ${ }^{6}$ no radica en su infrecuencia -es completamente habitual, por desgracia, este tipo de lances en el espacio universitario- sino en lo pionero de su desenlace: a raíz del proceso de reclamación administrativa que resolví iniciar, la universidad donde sucedió el acoso terminó por sancionar (con suspensión de empleo y sueldo7) por "falta muy grave de acoso laboral" a la persona ${ }^{8}$ perpetradora del acoso, funcionaria a la sazón y por entonces directora del departamento. Fue la primera que vez que dicha universidad impuso una sanción de este tipo. No hay tampoco noticia de que ninguna otra universidad española haya interpuesto nunca una sanción análoga, y aunque no existen datos ni estadísticas públicos contrastables al respecto, lo que obra en la línea de oscurantismo y la falta de transparencia que puebla el ámbito del acoso laboral y su persecución en la academia del siglo XXI, pese a las múltiples declaraciones y resoluciones públicas a favor de una ética contrastada de las instituciones universitarias.

Aquel reconocimiento pionero se logró, empero, tras más de dos años de durísima pugna social y administrativa que supusieron una importante victimización secundaria para mí misma. No en vano la lucha personal de una víctima de acoso laboral por su reconocimiento institucional ha sido definida por los expertos como la de David contra Goliat (Piñuel y Zabala, 2001), y una forma de revictimización constitutiva de daño fundamental, a menudo igual o mayor que la del propio acoso.

\footnotetext{
5 Como se ha indicado más arriba, se recuerda que la profundización en el caso y la etnografía son provisionales, de modo que aquí se resume los resultados preliminares y fundamentales del trabajo en curso.

6 Se toma la propia experiencia de la autora como "caso notorio" (hito) favorito, recordando a Bacon (en Benedict, 2006, p. 166).

7 El número de meses no se especifica expresamente por las cuestiones deontológicas referidas al respecto del anonimato; solamente se informa de que la estipulación de sanción por parte de la persona instructora del caso (reconocida penalista) se estimó en casi dos años completos, aunque se rebajó finalmente por la autoridad competente (el Rectorado), en forma de piedad institucional, a algo menos de un año completo, aunque manteniéndose siempre la tipificación de falta como "muy grave", ya que el acoso laboral se halla estipulado jurídicamente como tal.
}

8 Por las mismas razones aducidas en nota 6, se elude especificar el género de la persona sancionada. 
Otro elemento distintivo de interés del caso que nos ocupa es que sirvió para poner de manifiesto la vinculación sistémica entre la endogamia universitaria y el acoso laboral, a modo de relación inversa: ante situaciones donde estructuralmente se rompe la dinámica endogámica, suele subseguir un proceso de hostigamiento normalizado, que deviene fácilmente en acoso laboral, por parte de los estamentos que han visto roto el statu quo del vasallaje académico, y contra la "intrusa" o "extranjera" académica. Y todo ello, como se apuntaba en el proemio, sucede en los contextos de producción de conocimiento teórico sobre valores humanos como la libertad, la integración de la diversidad, la alteridad constitutiva del ser humano y la cultura, etc. Es precisamente sobre la tematización de esta aporía, de corte más filosófico y detenido, donde se halla el estadio en curso de esta investigación. Otros conceptos en tratamiento son las polisemias del dolor para la persona acosada, la honda dimensión anarcosindical de la lucha, las extensiones del vasallaje, los vericuetos de lo personal-político-académico, el cinismo institucional-corporativo frente a la ética personal y, finalmente, la dimensión fenomenológica de autoanálisis y la praxis política libertaria.

Se narra a continuación, de modo telegráfico, las principales líneas del caso. A inicios de la segunda década de este siglo, un departamento (ámbito de ciencias sociales) ofreció una plaza a concurso público y, como es habitual, su propio profesorado formaba parte de la comisión evaluadora. La plaza fue adjudicada a una persona que presentaba multitud de conexiones profesionales con una persona miembro de la comisión evaluadora (y del departamento, a la sazón), que acabaría siendo la persona declarada acosadora hacia mí. A este concurso, formalmente meritocrático, me había presentado yo misma y, tras la resolución a favor de aquella otra candidata, recurrí administrativa y judicialmente la decisión de la comisión, logrando un éxito pionero en este tipo de reclamaciones: por primera vez en la historia de aquel departamento, una persona "externa" ganaba un recurso contra la resolución de una plaza del propio departamento. Y allí, por tanto, me incorporé tras la definitiva adjudicación de esta plaza.

A lo largo de los distintos estadios judiciales de la reclamación por aquel puesto académico, se mantuvo la colaboración sin ambages del profesorado interno y, muy especialmente, aquella persona profesora que devendría acosadora, con la causa de la candidata externa que había "perdido" su plaza, desbancada por la candidata foránea que yo era. Esta colaboración, a raíz de la investigación posterior derivada en sanción, se ha probado como fraudulenta e ilegal, en un intento contrario a derecho de tratar que a toda costa ganara la candidata doméstica frente a la "intrusa", como se relata en la Resolución condenatoria.

Distintas formas crecientes de acoso laboral se fueron perpetrando en mi contra, en mayor medida y gravedad cuando la citada persona profesora ocupó el cargo en la dirección del departamento, hasta el punto de que resolví interponer una queja interna administrativa en la universidad, amparándome en un Protocolo para la Prevención y Respuesta ante el Acoso que recién se había estrenado. La reclamación pasó de ahí a la inspección de servicios, siendo estudiada y refrendada por tanto por varias instituciones y expertos, incluyendo la oficina del defensor universitario y la propia oficina contra el acoso; de ahí, se abrió un primer expediente de información reservada, instruido por un jurista experto en mobbing que igualmente acreditó la ocurrencia del mismo; y finalmente se logró la apertura del expediente disciplinario sancionador, cuya instrucción duró un año completo, que dio lugar a la citada sanción, todo ello regido por la regulación nacional correspondiente. Por los costes emocionales y de todo tipo, siempre rehusé recurrir a una vía judicial, incluso rechazando iniciar un litigio por daños y perjuicios contra la perpetradora ya existiendo sanción, y contra lo que me fue fuertemente recomendado por juristas expertos, que apoyaban la apertura de una causa en este sentido.

Más de dos años de pugna administrativa y académica supusieron en efecto tanto daño como el acoso en sí mismo, y ya que, pese a que el citado protocolo (en virtud de la Ley de Prevención de Riesgos) contemplaba la inmediata interposición de medidas de protección cautelar durante la investigación por mobbing, ninguna disposición de tipo alguno fue adoptada, pese a mis reiterados ruegos y peticiones, ni tampoco fui nunca peritada/auditada por el servicio de prevención de riesgos de la universidad. Todo ello supuso en suma un inmenso desgaste que ya está tipificado como victimización secundaria y que terminó por agotar mis fuerzas, hasta el punto de que, pese al éxito sin precedentes de mi reclamación, opté por solicitar un cambio de departamento para poder seguir desempeñando mi actividad profesional de un modo mínimamente normalizado, y ya que el entorno departamental no había cesado durante todo ese tiempo (y ante la inacción institucional) de continuar mostrando por múltiples vías la manifiesta hostilidad hacia mí (Crucio), a la vez que el apoyo incontrovertido y acrítico a la persona perpetradora, pese a tantas 
irregularidades (recuérdese las "maldiciones imperdonables" del universo potteriano, a modo de inicio retórico en el proemio de este texto, y como la Avada Kedavra, en realidad, se acaba de algún modo cumpliendo).

Estos apoyos citados se mostraron de forma interna, con medidas discriminatorias de diverso tipo hacia mí, y de forma externa, con manifestaciones de apoyo a la perpetradora ya incluso estando establecida la sanción: desde dimisiones solidarias con su causa, públicamente expresadas, desde cargos unipersonales hasta cartas de apoyo del instituto universitario al que pertenecía la persona perpetradora o incluso manifestaciones en redes sociales, insinuándose que era yo, en realidad, la verdadera acosadora; esta acusación en concreto fue emitida por el director de departamento que sucedió en su mandato a la persona acosadora, desde su perfil profesional en Facebook. De todo ello tuvieron noticia las autoridades académicas, manteniendo siempre un silencio que, según los estudios sobre acoso, deviene cómplice y revictimiza aún más a la víctima.

Así, durante todo el largo tiempo del litigio previo y las manifestaciones de apoyo posteriores a la persona sancionada por acoso, informadas por mí a las autoridades universitarias oportunas, la universidad no mostró ningún apoyo formal hacia mí, en incumplimiento de su propio protocolo y de la propia Ley de Prevención de Riesgos Laborales, a excepción de la gestión de facilitarme el cambio de departamento. Así, si bien la universidad mostró valentía y ética en la investigación del acoso en sí (bien que con mucha más dilación de lo que estipulan leyes y protocolos, y auspiciada/forzada por una intervención anarcosindicalista, con el subsiguiente desgaste para mí y como fue cotejado por la Inspección de Trabajo), no así se puede afirmar del cumplimiento de otras medidas ya estipuladas de protección y amparo a la víctima de acoso, que resultan imprescindibles si se desea evitar la victimización secundaria: cuando una víctima de acoso se decide a denunciar internamente, como fue el caso, se ve obligada a asumir una serie imponderable de daños morales y profesionales ulteriores, sin haber causado ella daño alguno.

Tal y como la doctrina jurídica ha venido configurando como elementos que se requieren para que se produzca mobbing, en el caso presentado pudieron ser documentalmente probados (de ahí el éxito inaudito de la reclamación), a saber:

1) Un elemento material consistente en la conducta de persecución u hostigamiento, sistemático y planificado e injustificado de un sujeto activo (compañero de trabajo, superior o subordinado) a un sujeto pasivo en el marco de una relación laboral. En este caso se pudo mostrar con pruebas que nunca se trató de un conflicto personal sin más entre dos personas, víctima y victimaria, que "se pelean" por así decir en igualdad de condiciones; antes bien, como se demostró, mi actitud (incluso ya interpuesta la sanción) fue siempre conciliadora (aunque asertiva con mis derechos), incluso en las situaciones más difíciles, mientras que por parte de la persona perpetradora se pudo demostrar actitudes hostiles manifiestas, sistemáticas y planificadas e injustificadas, incluso contrarias a norma y siempre por lo general, desde una posición de jerarquía o superioridad sobre mí. Resulta crucial cómo destacó la Resolución condenatoria al respecto, entre otros aspectos, la notoria normalización que el entorno acosador había realizado de todo tipo de conductas irregulares de la perpetradora.

2) Un elemento temporal o de habitualidad: la conducta hostil debe ser reiterada en el tiempo. Aunque los hechos sean leves aisladamente considerados, adquieren gravedad con la reiteración. Se excluyen los hechos esporádicos. Igualmente, todo ello fue documentalmente demostrable para el caso que nos ocupa.

3) Un elemento intencional. La conducta hostil debe ser intencionada o maliciosa. Se excluyen los hechos imprudentes o casuales. A este respecto, también se demostró a lo largo de la instrucción del caso la intencionalidad de estos actos acosadores fundamentada en el hecho probado de una clara animadversión de la victimaria hacia mi persona.

\subsection{Análisis de una pena en observación9: ostentando la alargada sombra del acoso}

La gravedad y la profunda afectación de las víctimas, en correlación con la responsabilidad incluso penal de los perpetradores, es comparada por los expertos con experiencias con la de una violación, un divorcio, la pérdida de un cónyuge, ir a una guerra o diferentes formas de tortura (Pheko, 2018); similar afectación, en resumen, que la de un síndrome de estrés postraumático severo (Harvey et al, 2009, p. 85). El suicidio

9 En deuda y recuerdo de la magnífica obra homónima de C. S. Lewis Una pena en observación, publicada en 1961. 
efectivo o, cuando menos, sus ideaciones, son frecuentes en los procesos graves de mobbing: el acoso desvirtúa y destruye la realidad social de la víctima a través de su aislamiento de una red laboral de relaciones con sentido, impidiendo o dificultando la realizacion de sus objetivos y limitando su confianza en la racionalidad del mundo social. Se fractura el autorespeto, se reduce el bienestar subjetivo y se constriñe la libertad (Nadja, 2013).

Estas consecuencias deletéreas para las víctimas han sido ya ampliamente descritas; en suma, se considera que la salud entendida del modo holístico defendido por la OMS (física, mental, social, familiar, incluso espiritual -que no necesariamente religiosa-) es dramáticamente comprometida y afectada en los casos de víctimas de mobbing, llegado con frecuencia a suponer cambios permanentes en la personalidad y, por supuesto, a afectar de forma radical a su entorno cercano, familiar especialmente.

Destaca sobre todo el miedo anticipatorio y la vergüenza profunda de estar siendo victimizada, lo que genera confusión sobre la aparente inhabilidad para luchar y protegerse a sí misma la víctima; estos sentimientos altamente destructivos son descritos en autoetnografías muy potentes de académicas que han padecido mobbing (Pheko, 2018, p. 7).

Los estudios han destacado la maliciosidad, con rasgos de personalidad de naricismo y violencia (no necesariamente física) en el perpetrador y aislamiento de la víctima (Kowal et al, 2018; Pheko, 2018), así como la frecuencia de intentar hacer pasar a ésta como mentalmente enferma o desequilibrada, irracional, etc. (Kowal et al, 2018, p. 6), sobre todo en la fase (si llega) en que la víctima decide afrontar a través de la confrontación y denuncia pública, quedando en evidencia el perpetrador y, caso, también los colaboradores silenciosos. Esto sucede tanto a nivel de reclamación administrativa como, por supuesto, a nivel judicial, incluso tras existir peritajes judiciales que acreditan de forma profesional que la dolencia de la víctima está ligada estructuralmente al acoso, además de haberse podido descartar simulaciones por métodos protocolizados (cf. González et al, 2008) ${ }^{10}$. Nuevamente, todo lo recién citado se cumple puntualmente en el caso que nos ocupa y afecta a mi propia persona como víctima de mobbing.

Frente a la manipulación con que clásicamente los perpetradores pretenden presentar la afectación de la víctima, culpándola por ello y con eso victimizándola aún más, es frecuente que las víctimas presenten un alto grado de defensividad en la negación de síntomas (González et al, 2008) o incluso el rechazo de tomar medicación, por no considerarse enfermos como tal sino discriminados por un entorno (ese sí) enfermo, lo que entraña una diferencia crucial.

De hecho, a mayor abundamiento, una de las diferencias tipificadas entre paranoia (de lo que el perpetrador y el entorno acosador suelen acusar a las víctimas) e hipervigilancia, lo muestra el hecho de las personas aquejadas de la primera suelan responder a la medicación en numerosas ocasiones, mientras que las personas en la segunda situación "[...] no aceptan fácilmente el tratamiento médico (salvo en circunstancias extremas). Este tipo de tratamiento raramente tiene efectos beneficiosos, e incluso a veces empeora a la persona" (Piñuel y Zabala, 2001, p. 97).

La calificación penal del mobbing en el caso de España solo data de 2010 pero, desde entonces, ha avanzado en jurisprudencia de un modo muy relevante y su consideración jurídica hoy no es inferior a la de otros tipos de acoso, como el sexual, si bien el imaginario popular todavía dista mucho de comprender estas formas de acoso como similares en su gravedad, agravio comparativo que resulta doblemente lesivo y victimizadora para las personas que padecen mobbing; en el presente caso, se me ha llegado a "afear" la comparación de la afectación del acoso laboral con otros, con expresiones del tipo "vas tú a comparar", incluso por parte de amistades y colegas cercanos. Frente a esta minusvaloración, como ha documentado la bibliografía especializada, a menudo el acoso laboral posee consecuencias incluso más negativas (efectos más graves y duraderos) para las víctimas que el acoso sexual (Hershcovis, Reich y Niven, 2015), seguramente porque el primero carece aún del respaldo legal (pese a su reconocimiento, la carga de la prueba aún recae exclusivamente en la denunciante, mientras que se reparte equitativamente en el caso del acoso sexual), y el reconocimiento sociales que, aunque poco a poco, el acoso sexual ya lleva aparejando largo tiempo:

10 Piñuel y Zabala (2001, p. 53) habla explícitamente del “[...] estigma y el prejuicio ya proyectado por el/los acosadores de "tener problemas de personalidad" o de "ser una persona conflictiva. La percepción pública de la persona acosada es así manipulada hábilmente por el grupo de acoso, de tal manera que se tiende a culpabilizar a la víctima de lo que le ocurre mediante atribuciones casuales creadas ad hoc". 
Analizando hasta un total de 12 variables, que incluyen entre otras, la satisfacción en el trabajo, el estrés laboral, los niveles de ira y ansiedad o el deseo de abandonar la empresa, hemos encontrado que todos estos sentimientos son mucho más negativos en las personas que están sufriendo acoso laboral que entre quienes sufren acoso sexual. (Hershcovis, $2008^{11}$ )

Todo ello, además, siendo el mobbing un proceso ya de por sí de dificilísima demostración; los expertos lo han llegado a calificar de "crimen limpio" o "perfecto", como se apuntó al inicio:

\begin{abstract}
Este tipo de violencia tiene la característica diferencial, respecto de otros tipos de violencia que se presentan en la empresa, de no dejar rastro ni señales externas, a no ser las de un deterioro progresivo de la víctima, que es maliciosamente atribuido a otras causas, como problemas de relación o de personalidad, carácter difícil, incompetencia profesional, etc. Se trata por ello de un "crimen limpio" del que no queda huella y en el que la carga de la prueba suele ser complicada y costosa. (Piñuel y Zabala, 2001, p. 56)
\end{abstract}

Finalmente, ha sido además aún más difícil esta contienda contra el acoso laboral, para mí, por carecer dicho combate del aurea épica, de la sensación de transformación y pulso epocal tan poderosos, que presentan otras luchas en otros espacios -sindicales, sexuales, selváticos... de tantas índoles-. Algo tan aburrido, tan soporíferamente burocrático (al menos en apariencia), como combatir el sistema haciendo escritos para una inspección de servicios o un defensor universitario, litigando con algo tan absolutamente prosaico y carente de romanticismo como es un acoso laboral... no, no resulta muy motivador para una activista social. No suena a barricada, es difícil inspirarse en cánticos y gestas cuando el propio oponente es tan insulsamente tedioso e invisible. Y, sin embargo, ahí estaba la arena política, ahí estaba la lucha en estos años.

Y ahí sigue.

5. Conclusiones: nombrando el acoso laboral

Sin un cambio de dioses, todo continúa como estaba.

(Antonio Machado)

La liquidez y la condición escurridiza del acoso laboral, como se ha visto, son ampliamente descritas en la literatura, adquiriendo ello una significación especial en el ámbito universitario que nos ocupa. A menudo las formas de acoso son tan sibilinas y sutiles, aunque no por ello menos dañinas (sobre todo en su mantenimiento en el tiempo) que pueden adoptar la forma de "Ignorarle o excluirle, hablando solo a una tercera persona presente, simulando su no existencia (ninguneándolo) o su no presencia física en la oficina o en las reuniones a las que asiste (“como si fuera invisible”)" (Piñuel y Zabala, 2001, p. 59). Según se recoge en la "Resolución del Parlamento Europeo sobre el Acoso Moral en el lugar del trabajo": "Sin embargo por regla general el acoso es de carácter más difuso, lo que dificulta la tarea de demostrar su existencia" (Anderson, 2001, p. 17). O bien:

El acoso laboral es mucho más sutil y es fácil que pase desapercibido para el resto de trabajadores. ¿Cómo demuestra un empleado ante su jefe que está siendo discriminado por sus compañeros, que le hacen el vacío a la hora de comer o que difunden rumores falsos sobre él? [...] A menudo, la única solución es abandonar la empresa, pero la autoestima, el estrés y otros problemas no se irán tan fácilmente. (Hershcovis, 2008 ${ }^{12}$ )

\footnotetext{
11 Sin paginación en línea.

12 Sin paginación en línea.
} 
Es evidente que la gravedad de esta afectación y de este mal radica en la relevancia sociopersonal de la dimensión laboral y la importancia del trabajo para la autoconcepción. De ahí la vinculación cada vez mayor de los estudios sobre mobbing con el elemento colectivo y de liderazgo, entre otros, y muy especialmente el moral (Pheko, 2018): la dimensión (in) ética del mobbing. Los elementos defendidos por autores como Taylor (en Nadja, 2013) como los ejes claves de la autonarración (cf. Cavarero, 2007) y, en definitiva, cruciales para la identidad, como la interpretación de la vida como buena y significativa (sentido moral intrínseco de la vida) o la dignidad, se radican de modo especial en el trabajo como actividad social y fuente elemental de autosentido, teniendo en cuenta la reciprocidad esencial de las relaciones humanas; así, el lugar de trabajo es fuente crucial de autonarración y ello, a su vez, recrudece lo que afecta el mobbing en su dimensión social y de ocurrencia en un espacio público.

La persona estigmatizada por ser víctima de mobbing atrae atención negativa del entorno, sobre todo cuando se decide a confrontar/denunciar, lo que conlleva una afectación significativa de la propia imagen (Pheko, 2018; Nadja, 2013), y resulta aún más significativo en el caso del acoso en la universidad, por cuanto la reputación académica tiene que ver con redes, vínculos, posibilidades y tantos imponderables que de manera informal se manejan y que afecta directamente a la carrera de las académicas. Esta afectación de la víctima, entre tantas otras, se vincula igualmente con esa atracción de atención negativa (publicidad negativa, se podría decir), que ha sido incluso llamada específicamente "mancha de aceite" (Piñuel y Zabala, 2001).

Nadja (2013) recuerda igualmente la lista de bienes primarios en relación a la pirámide de necesidades humanas ya clásica de Maslow en su teoría de la motivación, y en la que los bienes más importantes (la vida y unos ingresos suficientes para una existencia digna, el sentido de la justicia, la realización de objetivos, la racionalidad, el auto respeto basado en bases sociales, la libertad y la sensación de bienestar), se vinculan también de forma esencial con la dimensión laboral en nuestras sociedades. Desde la perspectiva contemporánea de la filosofía moral y la psicología, el acoso laboral es fuente de maldad moral porque priva a la víctima de bienes primarios que valorados en las sociedades liberales democráticas (Nadja, 2013).

Como se ha demostrado, los elementos fundamentales del acoso laboral como asunto de interés genérico e incluso de salud pública global son los siguientes:

- Su dimensión procesual: el acoso como proceso social.

- Su dimensión colectiva: se trata siempre de un proceso colectivo, social por ende, que requiere una atmósfera favorable para su cultivo, y en conexión con ello igualmente las soluciones y prevenciones propuestas tienen que ver también con la dimensión colectiva, con la atmósfera en general y el liderazgo en particular, aparte por supuesto de la penalización activa de los perpetradores concretos (no así de los "transeúntes", sobre los que la intervención debe ser de tipo más preventivista y formativo).

- Su dimensión (in) ética e (in) moral: la necesidad de articular una lectura de calado filosófico, sobre el fenómeno, desde la filosofía moral, ética y, más aún, política.

Así, todos los trabajos concuerdan en enfatizar el aspecto organizacional del mobbing, la importancia de la dimensión colectiva que tiene la atmósfera que es el caso de cultivo del mobbing, tanto para su atajamiento como para su prevención (Akella, 2016). Se destaca asimismo la necesidad de políticas organizacionales que penalicen claramente los comportamientos de acoso, así como incidan igualmente en los necesarios cómplices silenciosos y la mejora de regulaciones protocolizadas de protección de víctimas y sus intereses; en suma, remedios legales y tipificados porque tiene que ver con la dignidad (Akella, 2016), de un modo palmario.

Finalizo este artículo, tan personal como político, enfatizando la recomendación de que se continúe en la línea de mejorar e incrementar las dimensiones de liderazgo ético y los procedimientos de investigación sobre mobbing en nuestras instituciones, en general, y en la institución académica/universidad, en particular, para que no se sume, al daño producido por el acoso a la víctima, el estigma de una victimización secundaria, incluso terciaria, que puede terminar con su salida definitiva de la institución, mientras 
se sigue dando amparo, siquiera por omisión y por pura inercia conservadora, a los grupos de poder y "transeúntes" (Björklund et al, 2019, pp. 9ss), que consienten y validan este comportamiento inético esencial. Finalmente, para que aquellas tres maldiciones imperdonables no sigan siendo normalizadas en los ámbitos donde, precisamente, se ensaya, produce y publica ideológicamente su propia condena.

\section{REFERENCIAS}

Akella, D. (2016). Workplace bullying: not a manager's right?. SAGE, Journal of Workplace Rights, 1(10), 1-10. https://doi. org/10.1177\%2F 2158244016629394

Anderson, J. (2001). Resolución del Parlamento Europeo sobre el Acoso Moral en el lugar del trabajo. https://cutt. ly/ohbDocO

Bakan, I., Büyükbese, T., Ersahan, B. y Sözbilir, F. (2017). Ethical climate, job satisfaction and mobbing. The Journal of Turk \& Islam World Social Studies, 4(10), 92-132. http://dx.doi.org/10.16989/TIDSAD.177

Benedict, R. (2006). El crisantemo y la espada. Alianza Editorial

Björklund, Ch. et al (2019). Workplace bullying as experienced by managers and how they cope: a qualitative study of Swedish managers. International Journal of Environmental Research and Public Health, 16(23), 46-93. https://doi. org/10.3390/ijerph16234693

Cavarero, A. (2007). Tu che mi guardi, tu che mi racconti. Filosofia della narrazione. Feltrinelli Editore-Collana Elementi.

Deckers, J. (2020). The value of autoethnography in leadership studies, and its pitfalls. Philosophy of Management. https://doi.org/10.1007/s40926-020-00146-w

García Landa, J. A. (2007). Acoso laboral en la universidad. Vanity Fear, http://www.garciala.blogia.com

Giacalone R.A., Promislo M., Jurkiewicz C. (2016). Ethical Impact Theory: how unethical behavior at work affects individual well-being. En, A. (Coord.), Global Encyclopedia of Public Administration, Public Policy, and Governance. Springer. https://doi.org/10.1007/978-3-319-31816-5_2252-1

González, D., y Delgado, S. (2008). Informe pericial: un caso de acoso laboral. Revista de Psicología del Trabajo y de las Organizaciones, 24(1), 113-124. https://doi.org/10.4321/s1576-59622008000100006

Harvey, M., Treadway, D., Thompson Heames, J. \& Duke, A. (2009). Bullying in the 21st century global organization: an ethical perspective. Journal of Business Ethics, 85, 27-40. https://doi.org/10.1007/s10551-008-9746-8

Hershcovis, S. M. (2008, marzo 11). Entrevista. Reuters. https://es.reuters.com/article/entertainmentNews/ idESSAN14733020080311

Hershcovis, S. M., Reich, T. C. y Niven, K. (2015). Workplace bullying: causes, consequences, and intervention strategies. White Paper, International Affairs Committee of the Society for Industrial and Organizational Psychology. http:// www.siop.org/whitepapers/workplacebullyingfinal.pdf

Justicia, F. et al (2003). Informe sobre acoso laboral en la Universidad de Granada. Universidad de Granada. http://www. ugr.es/ ccoo/informeacosouniversidad.pdf

Justicia, F., Fernández de Haro, E., García Berbén, A. B., Villena Martínez, M. D., et al (2005). Acoso laboral en la universidad. Revista de Psicología General y Aplicada, 58(4), 473-484. https://dialnet.unirioja.es/servlet/ articulo?codigo $=1464944$

Kowal, J. y Sawicka-Gwiazda, G. (2019). Mobbing as a problem in management ethics. Annales. Etyka W Życiu Cospodarczym, 21(5), 131-145. http://doi.org/10.18778/1899-2226.21.5.11

Lee, C. (2019). Capturing the personal through the lens of the professional: The use of external data sources in autoethnography. Methodological Innovations, 12(1). https://doi.org/10.1177/2059799119825576

Leymann, H. (1996). Mobbing. La persécution au travail. Ediciones du Seuil

Muncey, T. (2005). Doing autoethnography. International Journal of Qualitative Methods, 4(1), 69-86. https://doi. org/10.1177/160940690500400105

Murphy, S. (2008). The role of emotions and transformational leadership on police culture: an autoethnographic account. International Journal of Police Science and Management, 10(2), 165-178. https://doi.org/10.1350/ ijps.2008.10.2.72

Nadja, M. (2013). Ethics of work and mobbing from the perspective of moral philosophy and psychology. Repozytorium Uniwersytetu Warszawskiego. pdf?sequence $=4$

Pheko, M. M. (2018). Autoethnography and cognitive adaptation: two powerful buffers against the negative consequences of workplace bullying and academic mobbing. International Journal of Qualitative Studies on Health and Well-Being, 13(1), 1459134. https://doi.org/10.1080/17482631.2018.1459134

Piñuel y Zabala, I. (2001). Mobbing. Cómo sobrevivir al acoso psicológico en el trabajo. Sal Terrae

Piñuel y Zabala, I., y Fidalgo, A. M. (2004). Cisneros scale to assess psychological harassment or mobbing at work. 
Psicothema 16(4), 615-624. http://www.psicothema.com/english/psicothema.asp?id=3041

Rojas-Solís, J. L., García-Ramírez, B., Bernardino, E. y Hernández-Corona, M. E. (2019). El mobbing en trabajadores universitarios: Una revisión sistemática. Propósitos y representaciones. Revista de Psicología Educativa, 7(3), 1-10. http://dx.doi.org/10.20511/pyr2019.v7n3.369

Rosander, M. y Blomberg, S. (2019). Levels of workplace bullying and escalation - a new conceptual model based on cut-off scores, frequency and self-labelled victimization. European Journal of Work and Organizational Psychology, 28(6), 769-783. https://doi.org/10.1080/1359432X.2019.164287

Soares, T. y Torga, E. M. M F. (2020) Assédio moral na pós-graduação: as consequências vivenciadas por docentes e discentes de uma Universidade Estadual brasileira. Arquivos Analíticos de Políticas Educativas, 28(11), 1-28. https://doi.org/10.14507/epaa.28.4883

Stouten, J., Baillien, E., Van den Broeck, A. et al. (2010). Discouraging Bullying: The Role of Ethical Leadership and its Effects on the Work Environment. Journal of Business Ethics, 95, 17-27. https://doi.org/10.1007/s10551-0110797-X

\section{AUTORA}

Amparo Saornil Comaposada. Se trata de un nombre ficticio que se ha optado para proteger la identidad de la autora, siguiendo pautas protocolares para estos casos.

\section{Conflicto de intereses}

Las autoras no presentan conflicto de interés posible.

Financiamiento

No existió asistencia financiera de partes externas al presente artículo.

Agradecimientos

A las camaradas anarcosindicalistas, por hacerlo posible, hoy y ayer. A las mías, luceros libertarios, por mantenerme en la vida. 